\title{
Pharmaceutical Health Outcomes and Policy Curricula Among Arab Countries: An Evaluation of 191 Academic Institutions
}

Eman Ali Alghamdi

Saudi Food and Drug Authority https://orcid.org/0000-0002-9727-6972

Omar M. Albalawi

Saudi Food and Drug Authority

Thamir M. Alshammari ( $\square$ Thamer.alshammary@gmail.com )

King Saud University https://orcid.org/0000-0002-5630-2468

\section{Research article}

Keywords: Arab countries, health outcomes, patient safety, pharmacoeconomics, pharmacoepidemiology, pharmacovigilance

Posted Date: October 14th, 2020

DOI: https://doi.org/10.21203/rs.3.rs-81389/v1

License: () (i) This work is licensed under a Creative Commons Attribution 4.0 International License. Read Full License 


\section{Abstract}

\section{Background}

Health outcomes and policies is a growing areas that is needed in the healthcare culture. This study aimed to examine the availability, extent and type of teaching curricula (pharmacoeconomics, pharmacoepidemiology, pharmacovigilance, patient safety) in Arab countries.

\section{Methods}

A retrospective observational study was conducted during the period of November 2019 to January 2020. The collection strategy was performed by listing of pharmacy colleges in each country and then reviewing the institutions' websites that met the inclusion criteria. Five criteria were utilized to assess the availability of these courses: full-time mandatory , full-time elective, partial mandatory, partial elective, and not offered.

\section{Results}

One hundred ninety-one academic institutions were screened during the study period. Of those, 151 (79.1\%) institutions were included in the study and their curricular descriptions were retrieved from their electronic websites. Pharmacoeconomics was the most taught subject among the four subjects. It was offered in 89/151 of the colleges among Arab countries. Among these 89 colleges that offered pharmacoeconomics, 74/89 offered it as a mandatory. While, Pharmacoepidemiology was offered in 51 colleges, with 44/51 offering the subject as a mandatory, and 6 out of those 44 list it as a full-time mandatory , while 38 out of those 44 offer it as a partially mandatory. Only 22 of the colleges offered pharmacovigilance, with 14/22 offering the subject as a mandatory. Finally, there was only one institution that offers the subject of patient safety. Moreover, most of the four courses investigated are only taught in the final two years of study.

\section{Conclusions}

The study found a clear shortcoming in the teaching pharmaceutical outcomes. More efforts are needed by academic institutions to review other institutions' curricula to ensure they meet the work needs and advanced pharmacy practice transformation.

\section{Keypoints}

- There is shortage in teaching the pharmaceutical health outcomes subjects among the universities in Arab countries.

- Pharmacovigilance and patient safety subjects were the lowest taught subjects compared to pharmacoeconomics and pharmacoepidemiology.

- Most of pharmaceutical health outcome subjects were taught in the last year of the college currucilum and not many of these sibjects have included in the internship year which is the training year.

- There is an urgent need to revise the current curriculum among the pharmacy colleges and perhaps other health colleges and include these subjects because of the need of healthcare system in specialized healthcare professionals in these subjects.

\section{Background}

Over the past few decades, there has been a substanitial increase in the number of schools that offer pharmacy programs in different Arab countries. Most of the pharmacy schools' curricula in these countries offer either five or six-year baccalaureate degree programs. The six-year program is called the "Doctor of Pharmacy Program (PharmD)", while the five-year program is called the pharmacy (PharmB) baccalaureate degree. Like western accredited programs, they are undergoing continuous change for the purpose of quality improvement and to meet local accreditation standards [1]. Increasingly, there is greater emphasis and demand on improved learning outcomes in the research on healthcare policy and outcome areas (e.g. pharmacoeconomics, pharmacoepidemiology, and pharmacovigilance) to prepare graduates to be more adaptable and better equipped to work in different environments and within interdisciplinary teams [2].

Initially, pharmacoeconomics was a sub-discipline of the field of health economics concerned with the analysis of pharmaceutical product costs and services in terms of their economic value [3,4]. The importance of pharmacoeconomic analysis to pharmacists relies on the viewpoints from which the analysis is conducted. There are two fundamental components of pharmacoeconomic analysis that will be considered in the pharmacoeconomic evalution: the first is the measure of the cost of pharmaceutical products, and the second is the measure of the outcomes, which are combined into a quantitiative measure or ratio [5] these two components are currently being used to make decisions in formulary management, disease management, and in the assessment of therapeutic and biotechnology drugs [6].

Pharmacoepidemiology is a discipline concerned with the use of drug therapy for disease treatment, which includes safety, efficacy, and effectiveness of the drug utilization rate [4]. Intense monitoring for safety is part of the study protocol in premarketing studies [7]. Post marketing surveillance is mainly based on the analysis of individual cases of adverse drug reaction (ADR) observations, or on the suspicion of ADR by healthcare providers. Thus,

pharmacoepidemiology is an essential discipline that should be integrated into an important analysis of information concerning areas such as adverse drug events and post-marketing surveillance research [9][10,11]. Besides that, pharmacoepidemiology has a vital role in building a knowledge base that supports the optimal use of medications and helps to make better informed drug therapy decisions, such as drug approval and drug withdrawal due to adverse events [12],[13]. Thus, a proper training has been highly needed in order for pharmacy professionals to be capable of understanding and utilizing fundamental pharmacoepidemiological concepts to appropriately evaluate the risk and benefit of drug therapy $[10,11,13]$. Thus, this indicates a need for pharmacoepidemiology training and education in pharmacy schools. Moreover, in 2006, the Accreditation Council for Pharmacy Education (ACPE) concluded 
that pharmacy programs in the US should provide their students with sufficient clinical epidemiological concept and a background in understanding biostatistics [14].

Pharmacovigilance is defined by the World Health Organization (WHO) as "the science and activities related to the detection, assessment, understanding and prevention of adverse drug reaction (ADR) or any other possible drug-related problems" [15]. The primary function of pharmacovigilance is to identify signals that may arise from one or several sources, including observational or experimental sources [16]. In addition, spontaneous ADR reporting is one of the most widely used tools of pharmacovigilance. The concept of spontaneous ADR reporting was initially developed in the 1960s after the thalidomide tragedy [17]. Since spontaneous ADR reporting depends on reports from health care providers (HCPs) and patients, the absence of these reports may lead to the failure of a spontaneous reporting system. However, the engagement of theses groups to take part in ADR reports is an ongoing challenge perhaps, perhaps due to failure to recognize an ADR or failure to report a recognized ADR. Likewise, pharmacists have a vital role in patient safety, starting from the creation of safe systems of medication distribution to working with patients to assure the delivery of optimal use. Nonetheless, pharmacists contribute to improving the quality of drug therapy through optimizing the organizational structures through which drug therapy is provided, especially by creating medication use systems and by regularly evaluating their performance [18]. Several researchers have examined the importance of teaching patient safety in medical education but not pharmacy education.

Therefore, these four disciplines (pharmacoepidemiology, pharmacoeconomics, pharmacovigilance, and patient safety) are emerging as important components of pharmacy education. The aim of this study was to investigate the level of teaching and the availability of teaching curricula, as well as the type and extent of the four subjects in the academic institutions of pharmacy colleges and undergraduate studies, among 22 Arab countries.

\section{Methods}

\subsection{Data Collection Strategy}

All member states of the Arab League were selected for review. The Arab League is a regional multi-national organization of Arabic-speaking countries on the African and Asian continents. There are 22 members of the Arab League, which include Algeria, the Kingdom of Bahrain, Comoros, Djibouti, Egypt, Iraq, Jordan, Kuwait, Lebanon, Libya, Mauritania, Morocco, Oman, Palestine, Qatar, the Kingdom of Saudi Arabia, Somalia, Sudan, Syria, Tunisia, the United Arab Emirates, and Yemen.[19-21] Data collection strategies were performed using a two-step process in the period from November 2019 to January 2020 . In the first step, a listing of pharmacy schools in each country was obtained from the Ministry of Higher Education and the International Pharmaceutical Federation (FIP)-World List of Pharmacy Colleges website in.[22] All academic institutions from each official website were identified and listed into Microsoft Excel spreadsheets for screening and selection. In the second step, a manual search was conducted in order to identify potential academic institutions that are related to the topic, yet unlisted. There was no restriction on language because we translated websites that were not available in either English or Arabic.

\subsection{Study Selection}

Two authors (EA, OA) independently reviewed each site of all participating academic institutions to ascertain which sites met the inclusion criteria. To be considered for inclusion, the studied Arab academic institutions required the availability of doctor of pharmacy degrees, or degrees in the pharmaceutical sciences. This includes both governmental and private academic institutions. As a result, all pharmacy colleges in these countries were listed and each institution's electronic websites were retrieved for in-depth investigation. This step was performed by the same two authors and included random checks by a primary author (TA) to ensure proper data selection.

\subsection{Data Extraction and Coding}

All included websites were searched by the authors (EA, OA) and formatted into Microsoft Excel spreadsheets (available from the primary reviewer on request). The data were extracted in standardized tables and verified by a primary author (TA) for accuracy and completeness. The list of all pharmacy college websites was deeply investigated and searched to examine the curricula description, especially the type and extent of these subjects. Five criteria were utilized to assess the availability of these subjects: 1) full-time mandatory courses, 2) full-time elective courses, 3) partially mandatory courses, 4) partially elective courses, and 5) courses that are not offered. Full-time courses means the subject is taught as the sole course, while partial time means it is part of another main course. The listed mandatory courses are core courses that will be listed in program requirements and is compulsory to meet the requirements of the program. The listed elective courses are courses that can be chosen from a list of optional courses recommended for students in a curriculum. The descriptive analyses were conducted using statistical analyses software (SAS ${ }^{\circledR} 9.4$, Cary, NC).

\section{Results}

A total of 191 academic institutions were identified by the electronic search conducted for this study. Of these, a curricular description was retrieved from their electronic websites for 151 institutions (79.1\%), while $40(20.9 \%)$ of the institutions had unavailabile websites and curricular descriptions that were not obtainable. This study showed that the highest number of pharmacy schools in this region were in Egypt (35; 18\%), Saudi Arabia (28; 15\%), and Iraq (21; $11 \%)$ (see Table 1).

\section{Table 1. Number of involved pharmacy colleges per country}




\begin{tabular}{|lll|}
\hline Country & No. of colleges/country $(\mathbf{N}=\mathbf{1 9 1})$ & No. of colleges with obtainable curricular/country $(\mathrm{N}=151)$ \\
\hline Egypt & 35 & 32 \\
\hline Saudi Arabia & 28 & 25 \\
\hline Iraq & 21 & 15 \\
\hline Sudan & 18 & 10 \\
\hline Jordan & 16 & 16 \\
\hline Syria & 15 & 13 \\
\hline Libya & 9 & 5 \\
\hline Algeria & 8 & 5 \\
\hline United Arab Emirates & 7 & 7 \\
\hline Yemen & 7 & 6 \\
\hline Morocco & 6 & 2 \\
\hline Palestinian Territories & 5 & 5 \\
\hline Lebanon & 5 & 3 \\
\hline Oman & 3 & 3 \\
\hline Somalia & 3 & 1 \\
\hline Bahrain & 1 & 1 \\
\hline Kuwait & 1 & 1 \\
\hline Qatar & 1 & 1 \\
\hline Tunisia & 1 & 0 \\
\hline Mauritania & 1 & 0 \\
\hline Comoros Island & 0 & 0 \\
\hline Djibouti & 0 & \\
\hline
\end{tabular}

Pharmacoeconomics was the most taught subject among the four subjects in the studied academic institutions. It was offered in $89 / 151$ (58.9\%) of the colleges among Arab countries. Among these 89 colleges that offered pharmacoeconomics, 74/89 (83\%) offered it as a mandatory course (40/74 as full-time and 34/74 as partial-time) (see Figure 1).

Pharmacoepidemiology on the other hand, was offered in $51(33.7 \%)$ colleges, with $44 / 51(86.3 \%)$ offering the subject as a mandatory course, with $6 / 44$ (13.6\%) offering it as a full-time mandatory course, while $38 / 44(86.4 \%)$ offer it as a partially mandatory course (See Figure 1$)$.

Only 22 (14.5\%) of the colleges offered pharmacovigilance, with 14/22 (63.3\%) offering the subject as a mandatory course, 12 of which list it as partially mandatory course, while two offer it as a full-time mandatory course (see Figure 1). There was only one academic institution identified that offers the subject of patient safety, and this was only a partially mandatory course. Moreover, most of the four courses investigated are taught only in the final two years of study.

As previously indicated, the vast majority $(35 / 191 ; 18 \%)$ of academic institutions that offer a pharmacy degree are in Egypt. Most of these academic institutions have the curricula provided on their website (32/35; $91 \%)$. Of these, only $15 / 32$ (46.8\%) colleges offer pharmacoeconomics, with $11 / 15$ (73\%) offering it as a mandatory course, six of which list it as a partially mandatory course, while five offer it as a full-time mandatory course. Furthermore, only nine of the colleges (28\%) offer pharmacoepidemiology, and this is mainly offered as a mandatory course (8/9; $88 \%)$. However, seven of the colleges have it listed in their curriculum descriptions as a partially mandatory course, while two offer it as a full-time mandatory course. The subject of pharmacovigilance is offered in seven (21.8\%) colleges. The majority of these offer patient safety as a partially mandatory course (6/7; $85.7 \%)$, and one offers it as a full-time elective course (see Figure 2).

Saudi Arabia stood out as being ranked the second country that offers a pharmacy program in the Arab League (28/191; 15\%), with the vast majority of these academic institutions providing curricular descriptions (25/28; $89 \%)$. Of these, the pharmacoeconomics subject is the most taught course in Saudi pharmacy colleges $(18 / 25 ; 72 \%)$, and most of these academic institutions offer it as a mandatory course $(17 / 18 ; 94.4 \%), 12$ of which list it as a partially mandatory course while five offer it as a full-time mandatory course. Furthermore, 15 of the 25 colleges $(60 \%)$ offer pharmacoepidemiology, and this is mainly offered as a mandatory course $(14 / 15 ; 93.3 \%)$, whereas 10 list it as a partially mandatory course and 3 list it as a full-time elective course. The subject of pharmacovigilance is the lowest offered course at Saudi pharmacy colleges $(6 / 25 ; 24 \%)$ compared to pharmacoeconomics (72\%) and pharmacoepidemiology (60\%). Of these, only three academic institutions offer pharmacovigilance as a mandatory course (only as partially mandatory). Furthermore, Saudi Arabia 
stood out as being the only country in the Arab League to offer the subject of patient safety in their pharmacy programs. The King Saud bin Abdulaziz University for Health Sciences (KSAU-HS) offers patient safety as a partially mandatory course tilted "Ethics \& Patient Safety" (See Figure 2).

Jordan is ranked among the top five countries in terms of the number of academic institutions $(16 / 191 ; 8 \%)$ that offer a pharmacy program in the Arab League. All 16 pharmacy colleges have their curricula available on their website. The majority of these colleges (13/16; 81.2\%) offer pharmacoeconomics, and most of these offer it as a mandatory course (12/13; $92.3 \%)$. Eight colleges list the subject as a full-time mandatory course. Also, only four of the sixteen total pharmacy colleges in Jordan offer pharmacovigilance (25\%) (two as a partially mandatory course and one as a full-time elective course), whereas only one college offers pharmacovigilance as a full-time elective (see Figure 2). In Iraq, 21 academic institutions offer a pharmacy program (21/191; 11\%), and of these 15 (71\%) provided a curricular description. However, the majority of these colleges (13; 86.4\%) offer pharmacoeconomics, and all list it as a full-time mandatory course, but none of these colleges offer pharmacoepidemiology, pharmacovigilance, or patient safety courses (see Figure 2).

Sudan was found to be among the top five countries in terms of the number of academic institutions $(18 ; 8 \%)$ that offer a pharmacy program in the Arab League. More than half of these colleges (10/18; 55.5\%) provided a curricular description. Of these, only three pharmacy colleges offer pharmacoeconomics and pharmacoepidemiology courses, and all of these listed academic institutions offer it as a mandatory course (3/10; $30 \%)$. Two of the three colleges taught these courses as partially mandatory courses, while one college offered both subjects as a full-time mandatory course (see Figure 2). Syria was also found to be one of the top countries in terms of the number of academic institutions $(15 ; 7 \%)$ that offer a pharmacy program in the Arab League, with the majority of colleges $(12 / 15 ; 10 \%)$ providing a curricular description. Of these, pharmacoeconomics courses were the most taught courses in pharmacy programs in Syrian pharmacy colleges (7/12; 58\%); five as partially mandatory courses $(71.4 \%)$, one as a full-time elective course, and one as a partially elective course. Furthermore, three of the twelve colleges offer pharmacoepidemiology (25\%), and this is mainly offered as a partially mandatory course in two colleges, although one of those colleges has it listed in their curriculum descriptions as a partially elective course (see Figure 2).

Nine colleges offer pharmacy programs in Algeria and eight colleges in Libya, and five of these provide curricular descriptions in each country ( $9 / 191 ; 4.7 \%$ and $8 / 191 ; 4.1 \%$, respectively). In Algeria, all these included institutions offer pharmacoepidemiology, however, only as a partially mandatory course (100\%). Furthermore, none of these offer pharmacoeconomics, pharmacovigilance, or patient safety. However, In Libyan colleges, none of the four investigated courses are offered.

In the United Arab Emirates, seven academic institutions were found that offered a pharmacy program (7/191; 3.6\%), all of which provided curricular description. Of these, the pharmacoeconomics courses were the most taught courses in pharmacy programs in Emiratis pharmacy colleges (6/7; 85.7\%), and most of these academic institutions offered it as a mandatory course $(5 / 6 ; 83 \%)$. Of these, three offered it as a full-time mandatory course, two colleges offered it as a partially mandatory course, and one listed it as a full-time elective course. Furthermore, four of the seven colleges offered pharmacovigilance (57\%), and this was mainly offered as a partially mandatory course in two colleges. Two out of seven pharmacy colleges listed pharmacoepidemiology in their pharmacy program (28.5\%), and both listed it as a partially mandatory course (see Figure 2). Similar to the United Arab Emirates, it was found that Yemen has seven academic institutions that offer a pharmacy program, and $(6 / 7 ; 85.7 \%)$ furthermore, they also provide their curricular description. Of those, only the University of Science and Technology offers two of the subjects investigated (pharmacoeconomics and pharmacoepidemiology; 14.2\%), and both listed them as partially mandatory courses (see Figure 2).

In Morocco, only two out of six established academic institutions offer a pharmacy program and provide a curricular description (6/191; 3.1\%); however, none of these colleges offer any of the four courses investigated. In the Palestinian Territories, all five pharmacy colleges in the state provide a curricular description of the pharmacy program $(5 / 191 ; 2.6 \%)$, with three colleges $(3 / 5 ; 81.2 \%)$ offering pharmacoeconomics, and only one listing it as a full-time mandatory course, while one lists it as a full-time elective and another lists it as a partially elective course. One out of the five pharmacy colleges listed pharmacoepidemiology in a pharmacy program offered subject (20\%) and only listed it as a full-time elective course. Furthermore, none of these collages offered pharmacovigilance or patient safety.

In Lebanon, there were five academic institutions that were found that offered a pharmacy program $(5 / 191 ; 2.6 \%)$, and three of those provided their curricular description $(3 / 5 ; 60 \%)$. Of these, all three pharmacy colleges offered pharmacoeconomics, and two out of three offered it as a full-time mandatory course while one offered it as a full-time elective course. Two pharmacy colleges listed pharmacoepidemiology $(2 / 3 ; 66.6 \%)$ as a mandatory course, one listed it as full-time, while the other college listed it as part of another mandatory course. Moreover, only one offered pharmacovigilance as a full-time elective course.

In Oman, all three pharmacy collages provided a curricular description $(3 / 191 ; 1.5 \%)$, and all of these pharmacy colleges offered pharmacoepidemiology (100\%); however, only as a partially mandatory course. Of these, only one pharmacy college offered pharmacoeconomics (33.3\%), which is offered by the University of Nizwa and taught as a partially mandatory course. However, none of these offered pharmacovigilance or patient safety.

In Somalia, three academic institutions offer a pharmacy program (3/191; 1.5\%). Of these, only Edna Adan University provided a curricular description (33.3\%), with pharmacoeconomics offered as a full-time mandatory course and pharmacoepidemiology (33.3\%) offered as a partially mandatory course.

Pharmacovigilance education has not yet been provided.

Finally, the remaining academic institutions offering a pharmacy program have been found in five countries (the Kingdom of Bahrain, Kuwait, Qatar, Mauritania, and Tunisia). Out of those countries, curricular descriptions were only obtainable in the Kingdom of Bahrain, Kuwait, and Qatar. The University of Bahrain offers a pharmacy program, and the courses of pharmacoeconomics, pharmacoepidemiology, and pharmacovigilance are all offered as electives. Kuwait has only one pharmacy program offered by the University of Kuwait; however, none of the four courses investigated in this study are taught. Qatar also only has one pharmacy program offered at the Qatar University, and only pharmacoeconomics and pharmacoepidemiology are offered, and these two subjects are integrated into one course "Pharmacoepidemiology\&Pharmacoeconomics" as a partially mandatory course. However, of the 22 members of the Arab League, Comoros and Djibouti are the only countries in which no pharmacy program is offered.

Page 5/10 


\section{Discussion}

This study examined the current situation of teaching health outcome research courses among 22 Arab countries. There are fairly satisfactory numbers in terms of pharmacy colleges among these Arab countries; however, unfortunately, two countries (Comoros and Djibouti) do not offer a pharmacy program, and this might affect both education and the healthcare system in these countries. Of the 22 Arab countries examined, 151 (79.1\%) university curriculums were retrieved from their electronic websites. Pharmacoeconomics was the most taught subject among the four subjects (89/151; 58.9\%), followed by pharmacoepidemiology $(51 ; 33.7 \%)$, and then pharmacovigilance $(22 ; 14.5 \%)$. Patient safety was offered in only one Saudi university, King Saud bin Abdulaziz University for Health Sciences (KSAU-HS). In comparison, there were only 126 parmacy programs offered in 2018 in the 22 Arab countries studied, whereas there now exist 191 schools, as discovered in this study.[22, 23] Most of all these pharmacy programs were established within the last 20 to 30 years.[1, 24, 25] While pharmacy education is undergoing global signficant transformation focused on advancing the practice of pharmacy education to improve health outcomes,[26, 27] many programs in Arab countries still continue to offer traditional pharmacy education with emphasis on drug compounding and preparation.

Over the last few decades, the discipline of pharmacoeconomics has become very important due to the new orientation towards considering the cost of drug therapy along with its safety and efficacy.[28] The results from the current study show that a higher number of pharmacy schools (89/151; 58.9\%) offer pharmacoeconomics education at the professional level when compared with 28/41 (68.3\%) from the survey conducted in 2013 by Qais Alefan.[29] The increase in the number of pharmacy schools offering pharmacoeconomics-related topics compared to 2013 could be due to the fact that 151 schools were reached by this study compared to only 41 schools that were reached in the study by Qais Alefan and colleagues in 2013. On the other hand, pharmacoeconomics education in the US is provided at almost all colleges and pharmacy schools.[30] According to the online retrieved curriculums, most of the included Arab countries located in North Africa (Morocco, Algeria, Libya) do not require schools to include pharmacoeconomic education at any level (partial/full or mandatory/elective). This may explain the low number of schools offering pharmacy baccalaureate programs and slo perhaps still utilizing the traditional pharmacy curriculum. However, the results of the current study show that, for the Eastern Mediterranean region, the majority of academic schools $88 / 130(68 \%)$ include pharmacoeconomic education, and $91 \%$ of pharmacoeconomic education is offered as a mandatory course. This is in accordance with the Accreditation Council for Pharmacy Education (ACPE) standards and guidelines that were set for professional pharmacy programs leading to a PharmD degree in the United States.[31]

Similarly, the majority of pharmacy colleges 27/38 (71\%) from the Gulf Cooperation Council (GCC) offer pharmacoeconomics education. These countries are in close proximity to each other and share similar cultural and societal characteristics. This might be due to the fact that most pharmacy schools and colleges in the GCC benchmarked the international curriculum, such as US, Australia, and Candada had done.[32] Also, this could be related to higher life expectancy rates and higher spending rates on the healthcare system which requires better models of economic spending.

This study reflects that there is still a deficit in the availability of pharmacoeconomics courses in pharmacy schools and colleges in the 22 Arab countries, and particularly in the Arab North African countries compared to US schools. The probable reason behind this is the fact that there are a low number of pharmacoeconomic experts and the fact that curriculum reviews were not comprehensive, as there was/is no involvement of pharmacy experts in health economics working in health sectors in developing pharmacy colleges curricula. Another issue might be the fact that pharmacoeconomics might not be a priority in the country's healthcare system, and finally, curriculum decision-makers (e.g., deans and staff) may lack the necessary awareness of the growing importance of pharmacoeconomics.[29]

In regards to the education of pharmacoepidimiology, in spite of the aforementioned fact that the ACPE concluded that pharmacy programs should provide their students with sufficient clinical epidemiological concepts and a background in understanding biostatistical analysis obtained from desined studies,[14] not many prior studies have examined the extent and level of pharmacoepidemiology teaching in pharmacy schools. This study shows that $51 / 151$ ( $33.7 \%$ ) of the included schools have provided pharmacoepidemiology education, which is a much lower number compared to the $74 / 89$ schools ( $83 \%$ ) from the survey conducted in 2007 in US.[33]

Several studies have reported on the importance of the pharmacoepidemiology discipline and that it be integrated into an important analysis of information concerning areas such as ADRs and post-marketing surveillance research. In addition, conducting research will be one of the main roles for pharmacists working in research units or departments in hospitals or other institutions, such as pharmaceutical companies and regulatory authorities. Therefore, there is a need for pharmacists to understand the concepts of pharmacoepidemiology. Furthermore, more extensive coverage of clinical epidemiology issues is needed to meet the demands of pharmacists, and should be conducted as responsible quantitative and qualitative research.

Furthermore, the WHO and FIB have reported the major role of pharmacists' engagement in the improvement of public health, health promotion and patient safety.[34] In response, there is a critical need for future pharmacists to understand and sensitize the comprehensive concepts of pharmacovigilance and patient's safety, which mainly emphasizes the improvement of patient health outcomes in either the community or hospital settings.

Several studies have examined pharmacists' knowledge, attitudes, and perceptions regarding their responsibilities toward pharmacovigilance; the results reported that pharmacists had a good attitude and were willing to participate in ADR reporting if they receive suitable education and training for ADR reporting systems and procedures.[35, 35, 36] As demonstrated by Hartman et al.,[37] the ways in which pharmacovigilance is taught differs according to country. In European countries, pharmacovigilance has been offered as integrated at an extensive level in therapeutic courses in undergraduate levels or programs, such as prescribing safety assessments (PSAs)[38] while in developing countries. Pharmacotherapy education still has its own challenges, and pharmacovigilance is less well established, both in general and in education.[39] A study conducted assessing the pharmacovigilance among the Arab countries revealed that variations in the maturity of pharmacovigilance systems and activities exist among Arabic countries. Countries such as Morocco, Tunisia, Saudi Arabia, Egypt, and Jordan have a mature pharmacovigilance system.[19] One of the major factors facilitating the establishement of mature pharmacovigilance systems is 
the targeting of education and training of HCP. Moreover, the WHO recommends that the importance of pharmacovigilance can be taught from the first year of education, since specific knowledge is required.[40]

Also, there is evidence that indicates that[41] the frequency of adverse events and medical errors are associated with the attitudes of healthcare professionals towards safety.[41] In this regard, a study was conducted in hospitals in different Arabic countries to assess the rate of ADR to patients in these countries. The results indicated that patient safety was a major concern for the health policy agenda in Arab countries and it was vital that the causes of harm to patients were identified and understood in order to develop strategies for improvement.[42] Therefore, improving patient safety culture should include all stakeholders, such as policymakers, healthcare providers, and those responsible for medical education. Francis Milligan[43] highlighted that healthcare curricula should be subjected to fundamental change to improve patient safety. This suggests that countries in the Arab world should focus on the need to provide training and education programs to healthcare professionals and students on the importance of systems in creating a culture of patient safety.

The consequences of ADRs have considerable importance in health economics. For instance, in Singapore, a study published in 2019 revealed that the economic burden of 81 admissions caused by ADRs cost US $\$ 570,404$. These costs were significantly higher for bleeding/elevated international normalized ratios compared to other ADRs, and for drugs acting on the blood coagulation system compared to other drug classes.[44] Similarly, in Europe in 2016, the estimated direct costs of ADR in hospitals for the public care sector ranged from $€ 2.8$ billion to $€ 84.6$ billion. [45] In 2012, Spanish authors conducted a systematic review and concluded that studies regarding ADR costs have heterogeneous designs. This study assessed the direct healthcare costs in hospitals and their results indicated that ADRs generate significant costs.[46, 47] For this reason, it is important to consider pharmacoeconomics, pharmacoepidimiology, pharmacovigilance, and patient safety education in undergraduate pharmacy colleges in order to improve patient outcomes and to assess the costs of harmful consequences, including ADRs, aimed to prevent or avoid this additional cost on the healthcare system. Furthermore, the cost analysis of ADRs may be useful in situations relating to decision making, such as post-marketing surveillance studies.

It has been previously argued that targeting undergraduate studies has several advantages for several reasons. Initially, it was thought that it was easier to acquire new skills in undergraduate study programs, and that the academic setting could provide a good environment to learn these disciplines. Pharmacy students usually work in a setting where education is part of their daily activities work on developing professional skills.[48, 49] However, if they have worked in clinical practice for years, it might be difficult to change their attitudes and behavior towards pharmacovigilance and patient safety. Therefore, greater attention should be given to training in these areas and in other medical schools.

The present study has some limitations. The data were collected from the online retrieved syllabi, not directly from the instructors. Also, this study was restricted to only undergrad pharmacy programs and not other graduate- and postgraduate-level education.

Findings from this study suggest that greater attention should be given to reviewing the curricula of pharmacy schools to consider pharmacoeconomics, pharmacoepidimiology, pharmacovigilance, and patient safety education in undergraduate pharmacy colleges in order to improve patient and healthcare outcomes. A unique opportunity exists for well-trained individuals to fill this gap, as providing these health outcome coursed for pharmacy students is especially important in an era that revolves around evidence-based healthcare decision making and preparing future pharmacist for different field of work.

The current study provides a basic understanding of the need for additional research among other pharmaceutical education institutions and healthcare faculties in the Arab countries, and the need for comparing results and conducting similar method-based studies among pharmacy colleges in different universities.

To the best of our knowledge, this is the first study that has evaluated the extent and level of the four disciplines (pharmacoeconomics, pharmacoepidimiology, pharmacovigilance, and patient safety education) that should be provided to pharmacy students in undergraduate pharmacy programs in the countries consisting of the Arab League. During the study period, there were 191 universities and colleges that provided approved pharmacy programs within pharmacy and medical schools, but only 151 schools were included.

\section{Conclusions}

The current study showed that teaching pharmaceutical outcomes and policy courses is still not common among the included Arab countries. It was foubd that pharmacoeconomics courses were the most offered courses among the four courses evaluated, and there is a great deficit in the availability of pharmacoepidemiology, pharmacovigilance, and patient safety education in pharmacy schools and colleges in the 22 Arab counties at the undergraduate level. More efforts are needed in order to review their curricula and ensure they meet the current work needed and to advance pharmacy practice transformation.

\section{Abbreviations}

ADR

Adverse Drug Reaction

WHO

World Health Organization

$\mathrm{HCP}$

Health Care Provider

FIP

International Pharmaceutical Federation

KSAU-HS

Page 7/10 
King Saud bin Abdulaziz University for Health Sciences

GCC

Gulf Cooperation Council

ACPE

Accreditation Council for Pharmacy Education

PSA

Prescribing Safety Assessments

\section{Declarations}

Ethics approval and consent to participate: Not applicable

Consent for publication: Not applicable

Availability of data and material: Not applicable

Competing interests: Authors have no conflict of interests to disclose

Funding: None

\section{Authors' contributions:}

EA played a role in the collecting the data, analyses and writing the manuscript .

OA played a role in the collecting the data, analyses and writing the manuscript .

TM finalized the manuscript, which ws susequently approved by all of the authors. Al authors read and approved the final manuscript.

Acknowledgements: None

\section{References}

1. Kheir N, Zaidan M, Younes H, El Hajj M, Wilbur K, Jewesson PJ. Pharmacy education and practice in 13 Middle Eastern countries. Am J Pharm Educ. $2008 ; 72$

2. Graber DR, Nappi J, Bellack JP, Lancaster C, Musham C, O’Neil EH. Curriculum topics in pharmacy education: current and ideal emphasis. Am J Pharm Educ. 1999;63:145-151.

3. Holdford DA. Pharmacoeconomics: From Theory to Practice. Am J Pharm Educ. 2010;74. https://www.ncbi.nlm.nih.gov/pmc/articles/PMC2865420/. Accessed 27 May 2020.

4. Briggs AH, Levy AR. Pharmacoeconomics and pharmacoepidemiology. Pharmacoeconomics. 2006;24:1079-1086.

5. Gattani SG, Patil AB, Kushare SS. Pharmacoeconomics: a review. Asian J Pharm Clin Res. 2009;2:15-26.

6. Reddy: Pharmacoeconomic education in US colleges... - Google Scholar. https://scholar.google.com/scholar_lookup? journal=Am+J+Pharm+Educ.\&title=Pharmacoeconomic+education+in+US+colleges+and+schools+of+pharmacy:+an+update\&author=M+Reddy\&author=1 5\&. Accessed 27 May 2020.

7. loannidis JP, Lau J. Improving safety reporting from randomised trials. Drug Saf. 2002;25:77-84.

8. Graham DJ, Waller PC, Kurz X. A view from regulatory agencies. Pharmacoepidemiology. 2000;:109-124.

9. Lawson DH. Pharmacoepidemiology: a new discipline. Br Med J Clin Res Ed. 1984;289:940.

10. Draugalis JR, Plaza CM. Emerging role of epidemiologic literacy. Ann Pharmacother. 2006;40:229-233.

11. Stewart RB. DRUG SURVEILLANCE-EPIDEMIOLOGY-ROLE OF THE CLINICAL PHARMACIST. Am J Pharm Educ. 1986;50:468-470.

12. Etminan M, Gill S, FitzGerald M, Samii A. Challenges and opportunities for pharmacoepidemiology in drug-therapy decision making. J Clin Pharmacol. 2006;46:6-9.

13. Hartzema AG. The beginnings of pharmacoepidemiology in The Annals. Ann Pharmacother. 2006;40:1647-1648.

14. ACPE Accreditation standards and guidelines for the professional program in pharmacy leading to their doctor of pharmacy degree (Appendix B. , III: American Colleges of Pharmacy Education; ). 2006.

15. WHO. The importance of pharmacovigilance. 2002.

16. Sciences C for IO of M, Sciences C for IO of M. Practical aspects of signal detection in pharmacovigilance: report of CIOMS Working Group VIII. Geneva Counc Int Organ Med Sci. 2010.

17. van Grootheest K, Olsson S, Couper M, de Jong-van den Berg L. Pharmacists' role in reporting adverse drug reactions in an international perspective. Pharmacoepidemiol Drug Saf. 2004;13:457-464.

18. Leape LL, Cullen DJ, Clapp MD, Burdick E, Demonaco HJ, Erickson JI, et al. Pharmacist participation on physician rounds and adverse drug events in the intensive care unit. Jama. 1999;282:267-270.

19. Alshammari TM, Mendi N, Alenzi KA, Alsowaida Y. Pharmacovigilance systems in Arab countries: overview of 22 Arab countries. Drug Saf. $2019 ;: 1-20$.

Page $8 / 10$ 
20. UMC | WHO programme members. https://www.who-umc.org/global-pharmacovigilance/who-programme-for-international-drug-monitoring/whoprogramme-members/. Accessed 30 Jun 2020.

21. Alshammari TM, Alenzi KA, Ata SI. National pharmacovigilance programs in Arab countries: A quantitative assessment study. Pharmacoepidemiol Drug Saf. 2020.

22. World List Of Pharmacy Schools. http://academic_institutional_membership.fip.org/world-list-of-pharmacy-schools/. Accessed 27 May 2020.

23. Al-Ghananeem AM, Malcom DR, Shammas S, Aburjai T. A call to action to transform pharmacy education and practice in the Arab world. Am J Pharm Educ. 2018;82.

24. Al-Wazaify M, Matowe L, Albsoul-Younes A, Al-Omran OA. Pharmacy education in jordan, saudi arabia, and kuwait. Am J Pharm Educ. $2006 ; 70$.

25. Sarheed O, Al-Azzawi AM, Nagavi BG. Pharmacy education in the United Arab Emirates. Am J Pharm Educ. 2014;78.

26. Alsharif NZ. Purposeful global engagement in pharmacy education. Am J Pharm Educ. 2017;81.

27. Anderson C, Brock T, Bates I, Rouse M, Marriott J, Manasse H, et al. Transforming health professional education. Am J Pharm Educ. 2011;75.

28. Kulkarni U, Dalvi K, Moghe VV, Deshmukh YA. Pharmacoeconomics: An emerging branch in health sciences for decision making. Afr J Pharm Pharmacol. 2009;3:362-367.

29. Alefan Q, Allmam S, Mukattash T, Mhaidat N, Alabbadi I, Rascati K. Pharmacoeconomics education in WHO Eastern Mediterranean region. Curr Pharm Teach Learn. 2015;7:819-825.

30. Makhinova T, Rascati K. Pharmacoeconomics education in US colleges and schools of pharmacy. Am J Pharm Educ. $2013 ; 77$.

31. Accreditation Council for Pharmacy Education. Accreditation standards and guidelines for the professional program in pharmacy leading to the doctor of pharmacy degree - Google Search.

32. Alkhateeb FM, Arkle S, McDonough SL, Latif DA. Review of national and international accreditation of pharmacy programs in the gulf cooperation council countries. Am J Pharm Educ. 2018;82.

33. Nwokeji ED, Rascati KL, Moczygemba LR, Wilson JP. Pharmacoepidemiology education in US colleges and schools of pharmacy. Am J Pharm Educ. $2007 ; 71$.

34. Federation IP. Good pharmacy practice. Joint FIP/WHO guidelines on GPP: standards for quality of pharmacy services. 2012.

35. Siddiqua A, Alshahrani N, lqbal JM, Ali AM, Abdulrahman R, Essa MAA, et al. Evaluation and assessment of the knowledge, attitude and practice of pharmacy students with respect to pharmacovigilance in a Saudi pharmacy school: a cross-sectional study. 2019.

36. Thomas D, Zachariah S. Knowledge, Attitude, and Practice of Pharmacovigilance in Developing Countries. In: Social and Administrative Aspects of Pharmacy in Low-and Middle-Income Countries. Elsevier; 2018. p. 177-193.

37. Hartman J, Härmark L, van Puijenbroek E. A global view of undergraduate education in pharmacovigilance. Eur J Clin Pharmacol. 2017;73:891-899.

38. Good medical practice. https://www.gmc-uk.org/ethical-guidance/ethical-guidance-for-doctors/good-medical-practice. Accessed 23 Jun 2020.

39. Abubakar AR, Chedi BA, Mohammed KG, Haque M. Perception of Nigerian medical students on adverse drug reaction reporting. J Adv Pharm Technol Res. 2015;6:154.

40. van Eekeren R, Rolfes L, Koster AS, Magro L, Parthasarathi G, Al Ramimmy H, et al. What future healthcare professionals need to know about Pharmacovigilance: introduction of the WHO PV Core curriculum for university teaching with focus on clinical aspects. Drug Saf. 2018;41:1003-1011.

41. Bonner AF, Castle NG, Men A, Handler SM. Certified nursing assistants' perceptions of nursing home patient safety culture: is there a relationship to clinical outcomes? J Am Med Dir Assoc. 2009;10:11-20.

42. Wilson RM, Michel P, Olsen S, Gibberd RW, Vincent C, El-Assady R, et al. Patient safety in developing countries: retrospective estimation of scale and nature of harm to patients in hospital. Bmj. 2012;344:e832.

43. Milligan FJ. Establishing a culture for patient safety-The role of education. Nurse Educ Today. 2007;27:95-102.

44. Chan SL, Ng HY, Sung C, Chan A, Winther MD, Brunham LR, et al. Economic burden of adverse drug reactions and potential for pharmacogenomic testing in Singaporean adults. Pharmacogenomics J. 2019;19:401-410.

45. Zsifkovits J, Zuba M, Geißler W, Lepuschütz L, Pertl D, Kernstock E-M, et al. Costs of unsafe care and cost effectiveness of patient safety programmes. 2016.

46. Allue N, Chiarello P, Bernal ED, Castells X, Giraldo P, Martínez N, et al. Assessing the economic impact of adverse events in Spanish hospitals by using administrative data. Gac Sanit. 2014;28:48-54.

47. Vallano AF, Agustí AE, Pedrós CX, de Bolós Arnau JM. Systematic review of studies assessing the cost of adverse drug reactions. Gac Sanit. 2012;26:277-283.

48. Maeda S, Kamishiraki E, Starkey J. Patient safety education at Japanese medical schools: results of a nationwide survey. BMC Res Notes. $2012 ; 5: 226$.

49. Nie Y, Li L, Duan Y, Chen P, Barraclough BH, Zhang M, et al. Patient safety education for undergraduate medical students: a systematic review. BMC Med Educ. 2011;11:33.

\section{Figures}




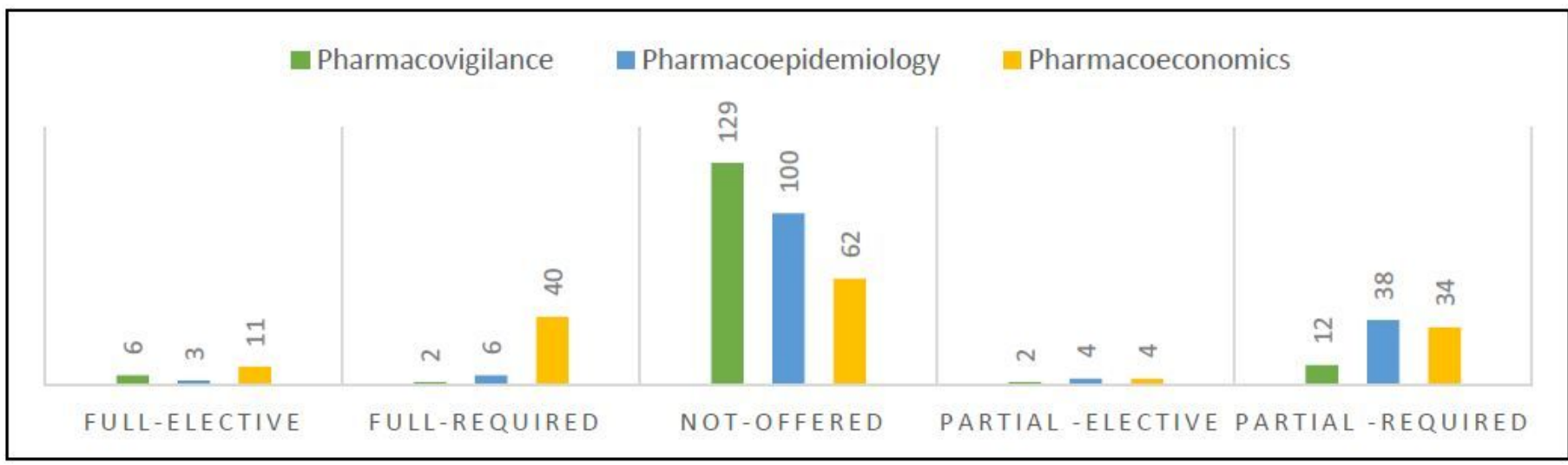

\section{Figure 1}

Type and extent of education of pharmacoeconomics, pharmacoepidemiology, and pharmacovigilance curricula per 151 colleges
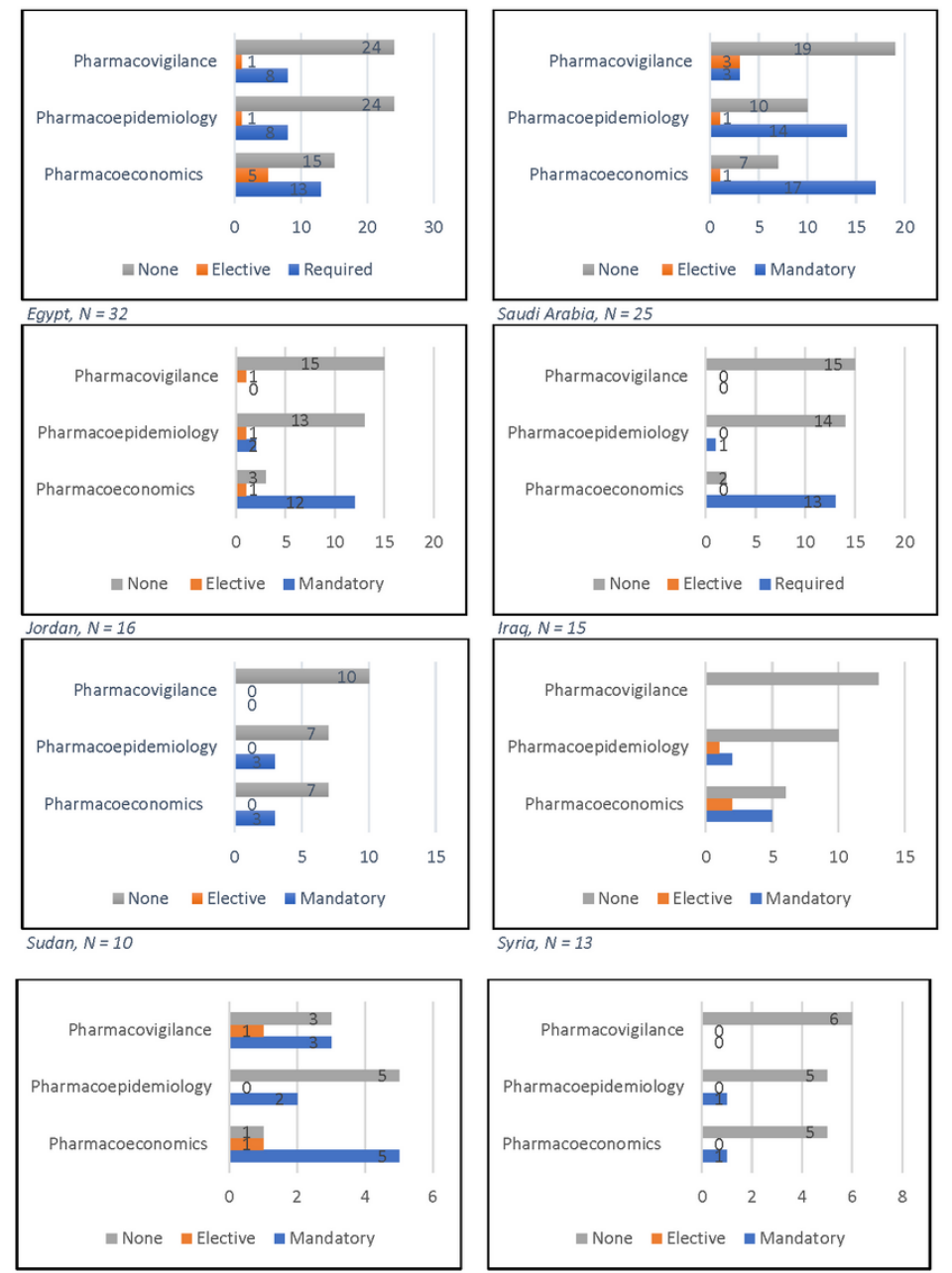

United Arab Emirates, $N=25$

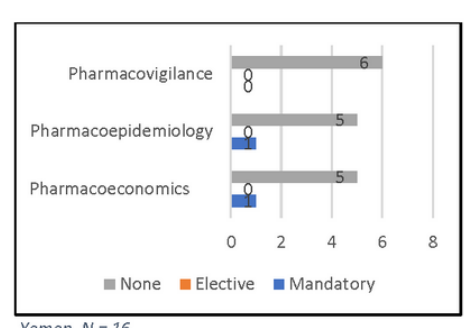

Yemen, $N=16$

Figure 2

Type and extent of education of pharmacoeconomics, pharmacoepidemiology, and pharmacovigilance curricula per contries 